\title{
Advances in Technology and Remote Cardiac Monitoring: Living the future of cardiovascular technology
}

\author{
Alaa Omar ${ }^{1}$ and Omar Lattouf ${ }^{2}$ \\ ${ }^{1}$ Mount Sinai Morningside Hospital \\ ${ }^{2}$ Emory University School of Medicine
}

September 25, 2021

\begin{abstract}
Hospital administrations and providers are more than ever in need for new technologies and innovative methods with clinical benefit at lower costs. Surgeons and clinicians depend on conventional risk stratification scores developed to allow physicians to establish the risk of perioperative mortality. However, the current practiced models of preventive cardiology largely depend on patient motivation and awareness to be able to apply such risk scores appropriately. It was not until the appearance of miniaturized pocket-sized, user-friendly digital technologies that the awareness started to grow, highlighting the importance of role of technology and artificial intelligence (AI) in modern day medicine.
\end{abstract}

\section{Advances in Technology and Remote Cardiac Monitoring: Living the future of cardiovascular technology}

Alaa Mabrouk Salem Omar, MD, MSc, PhD (1,3), Omar Lattouf, MD, PhD $(2,3,4)$

1) Department of Cardiovascular Medicine, Mount Saini Morningside, New York, NY, USA

2) Department of Cardiovascular Surgery, Mount Sinai Morningside, New York, NY, USA

2) Icahn School of Medicine at Mount Sinai, New York, NY, USA

3) Emory University School of Medicine, Atlanta, GA, USA

\section{Author of correspondence}

Omar Lattouf, MD, PhD

Professor of Cardiovascular Surgery

Department of Cardiovascular Surgery

Mount Sinai Morningside Hospital

111 Amsterdam Street, New York, New York 10025

Phone: 404-550-2076

Email: omar.lattouf@mountsinai.org

\section{Abstract}

Hospital administrations and providers are more than ever in need for new technologies and innovative methods with clinical benefit at lower costs. Surgeons and clinicians depend on conventional risk stratification scores developed to allow physicians to establish the risk of perioperative mortality. However, the 
current practiced models of preventive cardiology largely depend on patient motivation and awareness to be able to apply such risk scores appropriately. It was not until the appearance of miniaturized pocket-sized, user-friendly digital technologies that the awareness started to grow, highlighting the importance of role of technology and artificial intelligence (AI) in modern day medicine.

Key Words: Artificial Intelligence, cardiac surgery, outcomes, remote cardiac monitoring

The $21^{\text {st }}$ century has witnessed outstanding advances in medical diagnostics and interventional methods resulting in an exponential increase in medical data. Meanwhile, cardiovascular diseases remain a leading cause of death in the United States, claiming the lives of 17.9 million people yearly according to the World Health Organization (1) with annual costs of care that are projected to increase from $\$ 21$ to $\$ 53$ billion by the year 2030 (1). As such, hospital administrations and providers are more than ever in need for new technologies and innovative methods that can show clinical benefit and improve outcomes at lower costs. There are inherit challenges facing these technologies, however, as it is hard to expect when symptoms related to heart conditions will develop and who will develop complications of cardiac procedures and surgeries. Surgeons and clinicians depend on well-studied conventional risk stratification scores, such as EuroSCORE II, STS risk scores, which were developed to allow physicians to establish the risk of perioperative mortality (2). Recent studies, however, suggest that such scores, despite the improvements, tend to overestimate risk in the general population and underestimate it in high-risk populations; perhaps because these scores are built in a one-size-fit-all fashion ignoring the natural variability and heterogeneity (3). In addition, the current practiced models of preventive cardiology largely depend on patient motivation and awareness to be able to apply such risk scores appropriately. It was not until the appearance of miniaturized pocket-sized, user-friendly digital technologies that the awareness started to grow, highlighting the importance of role of technology and artificial intelligence (AI) in modern day medicine(4).

The applications of AI in our everyday life nowadays are various. From wearable devices to leadless pacemakers, holograms, robotic cardiac procedures, and transcutaneous techniques, the cardiovascular field is flooded with AI applications that has changed the language of clinical medicine quite considerably(4). Wireless devices, specifically, have invaded the medical field and are now integrated in the care of cardiac patients with the ability to remotely monitor various aspects of clinical and vital parameters. The widespread availability and use of cellular and wireless devices has grasped the attention of both the consumers and healthcare professionals. Millions of people nowadays use wearable devices such as Apple Watch to track their health, do simple diagnostic tests such as electrocardiographs and even alert emergency medical systems (EMS) in case of syncopal episodes or malignant ventricular rhythms (5). This consumer-level early warning network will likely play a big role in the coming years to automatically triage patients and help know when they should seek professional healthcare help.

Along these lines, Atılgan, et al., report in this issue of the successful use of a sophisticated method of remote monitoring of patients after having cardiac surgery. The device used incorporates measurements of clinical data such as blood pressure, heart rate, oxygen saturation, body temperature, blood glucose, as well as static and ambulatory electrocardiography [ECG] recording. Moreover, the system used, incorporated medication reminder, suggested daily life activities, diet and nutrition plans, and a video conference and communication platform. The use of this miniature method resulted in early detection of life-threatening events post-operatively, eliminated the need for multiple device monitoring after discharge, resulting in increasing patient's compliance, and was a successful aid towards appropriate hospitalization as well as avoidance of unnecessary hospitalizations.

The findings of the current study confirm that we are at the verge of a potential medical technological revolution. In a time of social distancing and no contact services, that the recent COVID-19 pandemic has cast over us, such a revolution appears to be more needed than ever. It is not a science fiction subject or an academic fantasy anymore. It is real and pivotal in our current practices to improve healthcare.

The technological applications discussed in the current study cannot be more relevant during this time of uncertainty. As such, one can now envision the future of cardiovascular medicine and surgery in a reduced 
contact environment from both a patient side and the healthcare provider side. This futuristic aspect of the relevance of this study are two folds; both have been developing with variable speeds, and presented herein in the forms of questions. The first is: how can we use the data retrieved from technological advances? The second is: , will the use the data itself, as novel diagnostic approach, uncover disease processes previously unseen?

The first aspect of this development is well represented in the study at hand in which impressive hardware and software technologies are developed with reported abilities to remotely and quasi-automatically collect, interpret and detect clinical abnormalities! These devices are programmed to operate semi automatically to gather and forward data to a monitoring center controlled by trained personnel.

The tele-health monitoring system, we are informed would automatically alert the responsible physicians, or his/her designee on worrisome developments.

As one discusses tele-health applications, one must bring Artificial Intelligence (AI) into focus, where AI applications are starting to exist and replace some tasks performed by healthcare specialists and technicians in both inpatient and outpatient settings. At this stage, AI applications using machine learning and deep learning algorithms promise exploration of new disease subclasses previously unseen to the human eyes. Efforts of operationalizing big data analytics towards disease phenotyping and better understanding the pathological and epidemiological heterogeneity are already underway $(3,6-10)$. This promises not only ability to collect data in an automated and remote fashion, as suggested in the current study, but also to automate and innovate diagnostics as well. However, several challenges do exist and must be addressed and resolved before AI-based diagnostic algorithms can be broadly applied in clinical practice. First, the development of reliable AI algorithm requires massive amounts of pre-labeled data for training computers in the quest for achieving human-level classification performance. The type and amount of data suitable for training such algorithms exist but frequently face the obstacle of healthcare privacy laws and medical data regulations making medical data less available compared to other fields of computer science. The development of a homogenous nationwide database using calibrated algorithms can be useful in that regard. Internet of medical things (IoMT) is a much-awaited promise that would take such solutions to an international level.

Furthermore, the rapid development of software and hardware biosensors, wearable monitors and implantable devices has not been accompanied by the same rate of development of the software that will be needed to manage the enormous amount of data generated from such devices. The ability to process massive data resulting from variabile of vendors, operators, software versions, and acquisition techniques can confound data processing. This will require tremendous bioinformatics capabilities to eventually understand and analyze such expansive amount of data.(11) (12)

In conclusion, the cardiac patient of the future, wired with a network of biosensors, wearable monitors and implantable miniature device, investigated and operated upon with robotic imaging arms and analyzed by computers will be extremely different from our current day-to-day patients. These biosensors will gather and transmit tremendous amount of personalized data and clinical responses to daily stimuli to personal smartphones powered to processes gathered data and direct the patient towards the next appropriate action or even do it independently. The future has already arrived, are we ready? (13-15)

\section{References:}

1. Levy D, Kenchaiah S, Larson MG, Benjamin EJ, Kupka MJ, Ho KK, et al. Long-term trends in the incidence of and survival with heart failure. N Engl J Med. 2002;347(18):1397-402.

2. Sullivan PG, Wallach JD, Ioannidis JP. Meta-Analysis Comparing Established Risk Prediction Models (EuroSCORE II, STS Score, and ACEF Score) for Perioperative Mortality During Cardiac Surgery. Am J Cardiol. 2016;118(10):1574-82.

3. Omar AMS, Krittanawong C, Narula S, Narula J, Argulian E. Echocardiographic Data in Artificial Intelligence Research: Primer on Concepts of Big Data and Latent States. JACC Cardiovasc Imaging. 2020;13(1 Pt 1):170-2. 
4. Bhattad PB, Jain V. Artificial Intelligence in Modern Medicine - The Evolving Necessity of the Present and Role in Transforming the Future of Medical Care. Cureus. 2020;12(5):e8041.

5. Isakadze N, Martin SS. How useful is the smartwatch ECG? Trends Cardiovasc Med. 2020;30(7):442-8.

6. Lancaster MC, Salem Omar AM, Narula S, Kulkarni H, Narula J, Sengupta PP. Phenotypic Clustering of Left Ventricular Diastolic Function Parameters: Patterns and Prognostic Relevance. JACC Cardiovasc Imaging. 2018.

7. Narula S, Shameer K, Salem Omar AM, Dudley JT, Sengupta PP. Reply: Deep Learning With Unsupervised Feature in Echocardiographic Imaging. J Am Coll Cardiol. 2017;69(16):2101-2.

8. Narula S, Shameer K, Salem Omar AM, Dudley JT, Sengupta PP. Machine-Learning Algorithms to Automate Morphological and Functional Assessments in 2D Echocardiography. J Am Coll Cardiol. 2016;68(21):2287-95.

9. Omar AMS, Narula S, Abdel Rahman MA, Pedrizzetti G, Raslan H, Rifaie O, et al. Precision Phenotyping in Heart Failure and Pattern Clustering of Ultrasound Data for the Assessment of Diastolic Dysfunction. JACC Cardiovasc Imaging. 2017;10(11):1291-303.

10. Salem Omar AM, Shameer K, Narula S, Abdel Rahman MA, Rifaie O, Narula J, et al. Artificial Intelligence-Based Assessment of Left Ventricular Filling Pressures From 2-Dimensional Cardiac Ultrasound Images. JACC Cardiovasc Imaging. 2018;11(3):509-10.

11. Blier L, Wolinski P, Ollivier Y. Learning with Random Learning Rates. arXiv preprint arXiv:181001322. 2018.

12. Lin M, Chen Q, Yan S. Network in network. arXiv preprint arXiv:13124400. 2013.

13. Krittanawong C, Zhang H, Wang Z, Aydar M, Kitai T. Artificial intelligence in precision cardiovascular medicine. Journal of the American College of Cardiology. 2017;69(21):2657-64.

14. Krittanawong C, Tunhasiriwet A, Zhang H, Wang Z, Aydar M, Kitai T. Deep learning with unsupervised feature in echocardiographic imaging. Journal of the American College of Cardiology. 2017;69(16):2100-1.

15. Picard MH, Adams D, Bierig SM, Dent JM, Douglas PS, Gillam LD, et al. American Society of Echocardiography recommendations for quality echocardiography laboratory operations. Journal of the American Society of Echocardiography. 2011;24(1):1-10. 